\title{
Available human feeder cells for the maintenance of human embryonic stem cells
}

\author{
Jung Bok Lee ${ }^{1,2}$, Ji Min Song ${ }^{1}$, Jeoung Eun Lee ${ }^{1}$, Jong Hyuk Park ${ }^{1}$, Sun Jong Kim ${ }^{1,2}$, \\ Soo Man Kang ${ }^{1}$, Ji Nie Kwon ${ }^{1}$, Moon Kyoo Kim ${ }^{2}$, Sung II Roh ${ }^{1}$ and Hyun Soo Yoon ${ }^{1}$ \\ ${ }^{1}$ Division of Stem Cell Biology, Medical Research Center, MizMedi Hospital 701-4, Kangseo-ku, Seoul 157-280, \\ Korea and ${ }^{2}$ Department of Life Science, College of Natural Sciences, Hanyang University, Seoul, Korea
}

Correspondence should be addressed to HS Yoon; Email: yoon@mizmedi.net

\begin{abstract}
Mouse embryonic fibroblasts (MEFs) have been previously used as feeder cells to support the growth of human embryonic stem cells (hESCs). In this study, human adult uterine endometrial cells (hUECs), human adult breast parenchymal cells (hBPCs) and embryonic fibroblasts (hEFs) were tested as feeder cells for supporting the growth of hESCs to prevent the possibility of contamination from animal feeder cells. Cultured hUECs, hBPCs and hEFs were mitotically inactivated and then plated. hESCs (Miz-hES1, NIH registered) initially established on mouse feeder layers were transferred onto each human feeder layer and split every 5 days. The morphology, expression of specific markers and differentiation capacity of hESCs adapted on each human feeder layer were examined. On hUEC, hBPC and hEF feeder layers, hESCs proliferated for more than 90, 50 and 80 passages respectively. Human feeder-based hESCs were positive for stage-specific embryonic antigen (SSEA)-3 and -4, and Apase; they also showed similar differentiation capacity to MEF-based hESCs, as assessed by the formation of teratomas and expression of tissue-specific markers. However, hESCs cultured on hUEC and hEF feeders were slightly thinner and flatter than MEF- or hBPC-based hESCs. Our results suggest that, like MEF feeder layers, human feeder layers can support the proliferation of hESCs without differentiation. Human feeder cells have the advantage of supporting more passages than when MEFs are used as feeder cells, because hESCs can be uniformly maintained in the undifferentiated stage until they pass through senescence. hESCs established and/or maintained under stable xeno-free culture conditions will be helpful to cell-based therapy.

Reproduction (2004) 128 727-735
\end{abstract}

\section{Introduction}

A human embryonic stem cell (hESC) line was first established by Thomson and his colleagues in 1998. They reported that inner cell masses (ICMs) of blastocysts proliferated continuously on mouse embryonic fibroblast (MEF) feeder layers. These cells showed specific characteristics including unlimited proliferation in an undifferentiated state, expression of specific markers, high level of telomerase activity and teratoma formation (Thomson et al. 1998).

Maintaining mouse ESCs in an undifferentiated state requires the use of leukemia inhibitory factor and/or mitotically inactivated MEF feeder layers (Evans \& Kaufman 1984, Smith et al. 1988, Willams et al. 1988, Roy et al. 2001). However, both feeder layers and basic fibroblast growth factor (bFGF) are needed to maintain the proliferation of hESCs (Thomson et al. 1998, Reubinoff et al. 2000, Park et al. 2003). MEF feeder cells have generally been used as feeder layers to support the unlimited growth of hESCs, but the use of animal feeder cells is associated with risks such as pathogen transmission and viral infection (Richards et al. 2002, Amit et al. 2003, 2004, Rosler et al. 2004). For these reasons hESCs cultured on animal feeder layers are not suitable for clinical applications. To overcome these problems, feeder layers comprising cells originating from human fetal and adult tissues have been tested. Some of the tested cells have allowed unlimited proliferation without differentiation, including human fetal skin fibroblasts, fallopian-tube epithelial cells (Richards et al. 2002), adult marrow cells (Cheng et al. 2003) and foreskin fibroblasts (Amit et al. 2003, Hovatta et al. 2003). Some of the human feeder cells supported the growth of hESCs in a similar way to MEF feeder cells. Richards et al. (2003) also reported the use of various primary cultured cells from human fetal, adult and neonatal tissues as feeder cells for supporting the growth of hESCs.

hESCs have also been cultured under serum- and/or feeder-free culture conditions. Xu et al. (2001) reported that hESCs are able to proliferate continuously on Matrigel 
(BD Biosciences, Bedfold, MA, USA) in MEF-conditioned medium (MEF-CM). Because extracellular matrices (ECMs) play multiple roles in the attachment, survival and growth of cells, Matrigel comprising laminin, collagen IV and heparan sulfate proteoglycan was used instead of MEF feeder layers. MEF-CM supporting the growth of hESCs in an undifferentiated state revealed the function of soluble factors secreted from MEF feeder cells. However, this culture protocol suffers from the need to maintain MEF cells in order to obtain MEF-CM and from the fact that Matrigel is also an animal product. There are some additional reports of hESCs grown under serum- and/or feeder-free culture conditions using feeder-CM or a growth cocktail (Amit et al. 2004, Carpenter et al. 2004, Rosler et al. 2004). Although hESCs maintained in these conditions exhibited typical morphology, marker expression and differentiation capacity, little is understood about the factors that regulate permanent growth without differentiation.

This study tested the use of three types of human cell (derived from uterine endometrium (hUECs), breast parenchyma (hBPCs) and abortus (hEFs)) as new feeder layers for supporting the unlimited growth of hESCs, and examined the characteristics of hESCs maintained on each human feeder layer.

\section{Materials and Methods}

\section{Isolation and culture of human primary cells}

All tissues used in this study were obtained from patients following benign disease hysterectomy or benign neoplasia or therapeutic abortion. Six patients participated in this study (patients' were aged: 34-38 years for endometrial tissue; 26-34 years for breast tissue; 32 years for aborted fetus). The institutional review board approved the study and informed consent was obtained from all patients. All the tissue samples were obtained from clinical biopsy specimens. hUECs were isolated using previously described methods (Telgmann et al. 1997, Apparao et al. 2002). Briefly, endometrium was finely minced and enzymatically digested with $0.25 \%(\mathrm{v} / \mathrm{v})$ collagenase $\alpha$ (Sigma) and $10 \mathrm{U} / \mathrm{ml}$ DNAse I (Sigma) in Dulbecco's modified Eagle's medium (DMEM)/F-12 (Gibco Invitrogen). Primary hBPCs were isolated from normal human breast tissues as previously described (Hahm \& Ip 1990, Ricciardelli et al. 2002). In the process of hBPC isolation, collagenase IV was replaced by collagenase A (Roche) and hEF cells were isolated using a mechanical procedure only (Van Antwerp et al. 1996, Lee et al. 2000). The internal organs (e.g. intestine, liver and heart) were first removed from 13-week-old fetuses; organs were then homogenized into small pieces by gentle pipetting. Cells that were enzymatically or mechanically dissociated from each tissue were then plated onto culture flasks and prolonged culture was performed using DMEM/F-12 supplemented with $10 \%(\mathrm{v} / \mathrm{v})$ fetal bovine serum (FBS; Hyclone, Logan, UT, USA), $4 \mathrm{mM}$ glutamine (Gibco
Invitrogen), $20 \mathrm{mM}$ HEPES, $100 \mathrm{U} / \mathrm{ml}$ penicillin and $100 \mu \mathrm{g} / \mathrm{ml}$ streptomycin (Sigma). The cells were cultured until they passed through senescence. Cultured human primary cells were tested for the presence of infection by mycoplasmas and various viruses.

\section{Preparation of feeder layers}

Cultured primary human cells from each tissue were used as feeder cells from passage 3 . These cells were mitotically inactivated using $10 \mu \mathrm{g} / \mathrm{ml}$ mitomycin C (Sigma) for $1.5 \mathrm{~h}$ and then washed three times with PBS. These cells were detached with $0.05 \%(\mathrm{v} / \mathrm{v})$ trypsin and $0.53 \mathrm{mM}$ EDTA (Gibco Invitrogen) and washed three times by centrifugation and resuspension with DMEM/F-12 medium. Dissociated cells were counted using a hemocytometer and were plated with DMEM/F-12 medium supplemented with $10 \%(\mathrm{v} / \mathrm{v})$ FBS onto $0.1 \%(\mathrm{v} / \mathrm{v})$ gelatin-coated fourwell plates at $8.0 \times 10^{4}$ cells/well. After the attachment of plated cells, the medium was changed to DMEM/F-12 supplemented with $20 \%(\mathrm{v} / \mathrm{v})$ knockout serum replacement (SR) (Gibco Invitrogen), $1 \mathrm{mM}$ glutamine, $0.1 \mathrm{mM} \beta$ mercaptoethanol (Sigma), 1\% ( $/ \mathrm{v})$ non-essential amino acids and $4 \mathrm{ng} / \mathrm{ml}$ bFGF (Gibco Invitrogen).

\section{hESC culture on human feeder layers}

Miz-hES1 (NIH registered) was first established on mouse feeder layers. At passages 35, 50 and 30, hESCs maintained on MEF feeder layers were transferred onto hUEC, hBPC and hEF feeder layers respectively. hESCs cultured on each human feeder layer were passaged every 5 days by mechanical harvesting. About $4-5 \mathrm{~h}$ before the splitting of hESCs, the medium (DMEM/F-12 supplemented with $10 \%$ FBS) used to plate feeder cells was replaced by the medium for the culture of hESCs (DMEM/F-12, 20\% $\mathrm{SR})$, followed by a washing step. Prolonged culture of hESCs was performed at $37^{\circ} \mathrm{C}$ in $5 \% \mathrm{CO}_{2}$, with the culture medium being changed every day.

\section{Characterization of hESCs adapted on human feeder cells}

The morphology, expression of specific markers and differentiation capacity of hESCs cultured on each human feeder layer were examined. Cell morphology was observed under an inverted microscope every day and expressions of pluripotent cell-specific markers were tested immunocytochemically using stage-specific embryonic antigen (SSEA)-1 and -4 (Hybridoma Bank, University of lowa, IA, USA), tumor rejection antigen (TRA)-1-81 (Chemicon, Temecula, CA, USA) and APase. Immunocytochemistry was performed as described by Park et al. (2003). Briefly, for APase staining, cultured cells were fixed by $4 \%(\mathrm{v} / \mathrm{v})$ paraformaldehyde, permeabilized by $0.2 \%(\mathrm{v} / \mathrm{v})$ Triton $\mathrm{X}-100$ and stained using a kit containing 1 nitro blue tetrazolium chloride/S-bromo-4-chloro-3-indolyl phosphate (NBT/BCIP) as the substrate (Roche). In the process 
of SSEA-1 and -4 and TRA-1-81 staining, fixed cells were incubated with each primary antibody; antibody localization was performed using rabbit anti-mouse immunoglobulin secondary antibodies conjugated to fluorescein isothiocyanate or tetramethylrhodamine isothiocyanate (Jackson ImmunoResearch Laboratories, West Grove, PA, USA). Nuclei of each human feeder cell and hESC were visualized by the staining of Hoechst 33258 .

To analyze the karyotypes of hESCs cultured on each human feeder cell, cell division was blocked by $0.1 \mu \mathrm{g} / \mathrm{ml}$ colcemid (GibcoBRL/Invitrogen) in metaphase for $1-2 \mathrm{~h}$. Cells were then trypsinized and resuspended in hypotonic $\mathrm{KCl}$ solution (Sigma), incubated for 20 minutes at $37^{\circ} \mathrm{C}$ and fixed with 3:1 methanol:acetic acid. Chromosomes were visualized using G-band staining. More than 100 cells were examined in this way.

The differentiation of hESCs maintained on human feeder layers was indicated by the formation of teratomas (in vivo) and embryoid bodies (EBs, in vitro). Mechanically dissociated hESC colonies were injected into the right testes of severe combined immune deficiency (SCID)-beige mice and PBS without hESCs was injected into the left testes as a negative control. Twelve weeks after the injection, teratomas were observed with hematoxylin-eosin staining. In vitro differentiation of hESCs was confirmed by the formation of EBs. To form EBs, feeder cells were removed and hESC colonies were harvested. Harvested colonies then transferred to EB culture medium (DMEM/F-12, 20\% SR without bFGF), and the culture continued. After 2 weeks of culture, expressions of tissue-specific genes were examined by RT-PCR to confirm their differentiation capacity. Marker primers were used to test differentiation of hESCs into derivates of three embryonic germ layers: ectoderm (NF-68: forward, acgctgaggaatggttcaag; reverse, tagacgcctcaatggttcc; keratin: forward, aggcccaatacgaggagatt; reverse, atagccactggagatggtgg), mesoderm (enolase: forward, gttcaatgtcatcaatggcg; reverse, gtgaacttctgccaagctcc; cartilage matrix protein (CMP): forward, aaaaagggcaatgacaccag; reverse, ttgtgcagtctctgaggtgg; kallikrein: forward, gctttctcagccaggacatc; reverse, tattctttgcctcccaggtg; cardiac actin (cATC): forward, tatttgctcccttgcttgga; reverse, cctaccccaaaaacaaacga; $\delta$-globin: forward, catggtgcatctgactcctg; reverse, gtacttgtgagccagggcat; $\beta$-globin: forward, catggtgcatctgactcctg; reverse, gccaccactttctgataggc) and endoderm (albumin: forward, cttcctgggcatgttttgt; reverse, ggttcaggaccacggataga; $\alpha 1$-antitrypsin $(\alpha 1-\mathrm{AT})$ : forward, actgtcaacttcggggacac; reverse, ccccattgctgaagacctta; PDX-1: forward, agctttacaaggacccatgc; reverse, ttcaacatgacagccagctc; insulin: forward, ccatagtcaggagatgggga; reverse, gctggtagagggagcagatg; $\alpha$-fetoprotein $(\alpha-\mathrm{FP})$ : forward, tgaaaaccctcttgaatgcc; reverse, tcttgcttcatcgtttgcag). Oct-4 (forward, aagaacatgtgtaagctgcggccc; reverse, ggaaaggcttcc ccctcagggaaagg) and $\beta$-actin (forward, atctggcaccacaccttctacaatgagctgcg; reverse, cgtcattactcctgcttgctdatccacatctgc) were used as negative and positive control.

To analyze DNA contents of feeder cells and hESC colonies cultured on human feeder layers were harvested by trypsin-EDTA (Sigma) and $0.1 \%(\mathrm{v} / \mathrm{v})$ collagenase type IV (Sigma); hESCs colonies were dissociated into single cells by treatment with $0.5 \mathrm{mM}$ EDTA. Cells washed with PBS were fixed by $70 \%(\mathrm{v} / \mathrm{v})$ ethanol for more than $1 \mathrm{~h}$ at $4{ }^{\circ} \mathrm{C}$ and washed thoroughly. To analyze the DNA content of hESCs, fixed cells were stained with $100 \mu \mathrm{g} / \mathrm{ml}$ propidium iodide (Sigma) containing $100 \mu \mathrm{g} / \mathrm{ml}$ Rnase and were measured by flow cytometry (EPICS ALTRA, Beckman Coulter, Miami, FL, USA). Proliferation of hESCs was also evaluated by using a 5-bromo-2'-deoxyuridine (BrdU) incorporation kit according to the manufacturer's
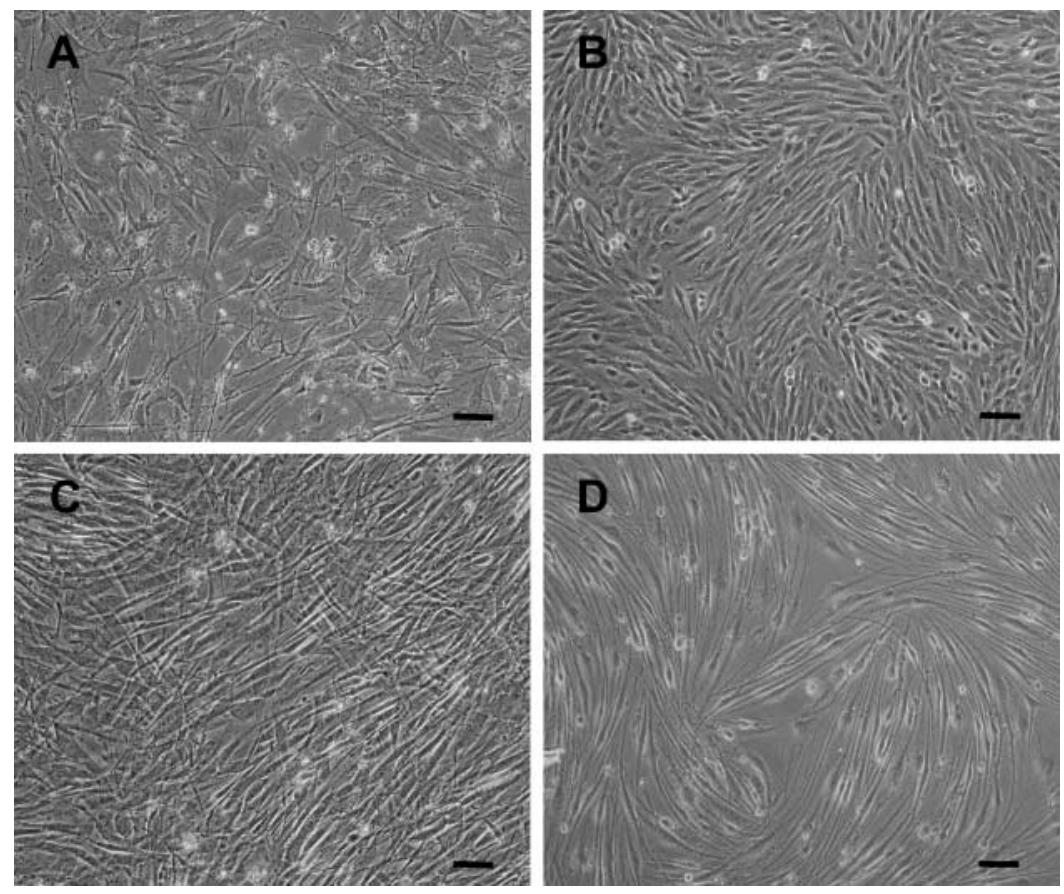

Figure 1 The morphologies of various feeder cells. (A) MEF cells from CF-1 mice, (B) hUECs at passage 18, (C) hBPCs at passage 13 and (D) hEFs at passage 7. Each feeder cell showed different morphology. Scale bars represent $100 \mu \mathrm{m}$. 
instructions (Roche). Briefly, hESCs were cultured in serum-starved medium (DMEM/F-12 supplemented with $0.1 \% \mathrm{SR}$ ) to synchronize cell cycle in the $\mathrm{G}_{1}$-stage for $36 \mathrm{~h}$; they were then transferred to fresh medium $(20 \%$ $\mathrm{SR}$ ) and BrdU labeling solution for $4 \mathrm{~h}$. Incorporated BrdU was localized using anti-BrdU working solution and was measured by ELISA reader (Microplate Manager; BioRad). Statistical analysis was performed using Student's $t$-test.

\section{Results}

\section{Culture of human primary cells}

Isolated and cultured primary human cells from uterine endometrium, breast parenchyma and embryonic fibroblasts were continuously grown and split for about 25 passages. Because these cells exhibited a similar proliferation rate, cultured cells were split every 7 days using enzymatic dissociation. However, the cells showed differing morphology (Fig. 1). For the long-term use of these cells as feeder cells, these primary cultured cells were cryopreserved in early passages, and we tested whether frozen-thawed cells maintained a similar morphology and proliferation rate. Neither primary nor frozen-thawed cells were infected with any viruses or mycoplasmas (data not shown).

\section{Morphology of hESCs}

hESCs transferred onto the three different human feeder layers maintained continuous proliferation. Miz-hES1 cells maintained on hUEC, hBPC and hEF feeder layers were
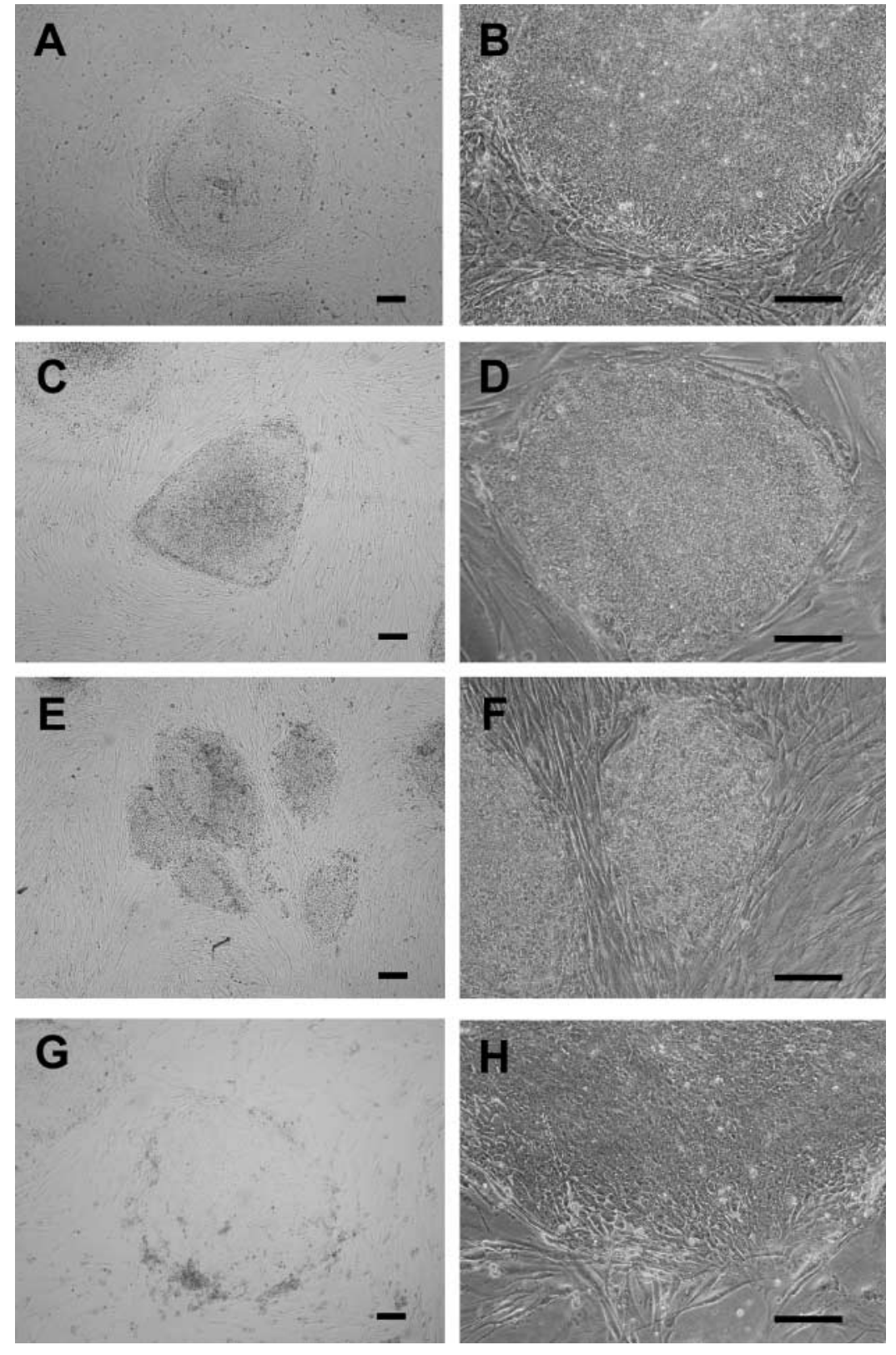

Figure 2 The morphology of hESC colonies cultured on MEF, hUECs, hBPCs and hEFs. hUEC- (C and D) and hEF-based ( $\mathrm{G}$ and $\mathrm{H})$ hESC colonies were thinner and flatter than MEF- (A and B) and hBPC-based (E and F) hESC colonies. Phase-contrast photographs (B, D, F and $\mathrm{H}$ ) showed typical hESC morphology. Scale bars represent $200 \mu \mathrm{m}(\mathrm{A}, \mathrm{C}, \mathrm{E}$ and $\mathrm{G})$ and $100 \mu \mathrm{m}(\mathrm{B}, \mathrm{D}, \mathrm{F}$ and $\mathrm{H})$. 
cultivated for over 90, 50 and 80 passages respectively. hESCs maintained on MEF feeder layers exhibited typical morphology such as a high nucleus-to-cytoplasm ratio, one to three prominent nucleoli and close spacing between the cells. In contrast, the morphology of hESCs maintained on hUEC and hEF feeder layers was slightly different from those based on MEF or hBPC feeder layers. The hUEC- and hEF-based hESCs were thinner and flatter than MEF- or hBPC-based hESCs and the spacing between cells was also greater. hBPC-based hESC colonies had a higher density than the other feeder-based hESCs (Fig. 2).

\section{Expression of pluripotent cell-specific markers}

While SSEA-1, which was used as negative control, was not detected in any of the hESC colonies cultured on human feeder layers (data not shown), the expressions of APase (used as a marker of continuously proliferating cells), SSEA-4 and TRA-1-81 were strongly positive in all hESC colonies based on human feeder layers, as was the case in MEF-based hESCs (Fig. 3). Staining of pluripotent cell-specific markers, performed every ten passages, revealed that all hESCs maintained on human feeder layers maintained their expression of pluripotent cellspecific surface markers.

\section{Karyotype analysis}

Karyotype analysis was performed with hESCs expanded on each human feeder cell. Miz-hES1 cultured on hUEC (Fig. 4A), hBPC (Fig. 4B) and hEF (Fig. 4C) showed normal 46, XY karyotypes. Karyotype analysis was performed every 6 months.
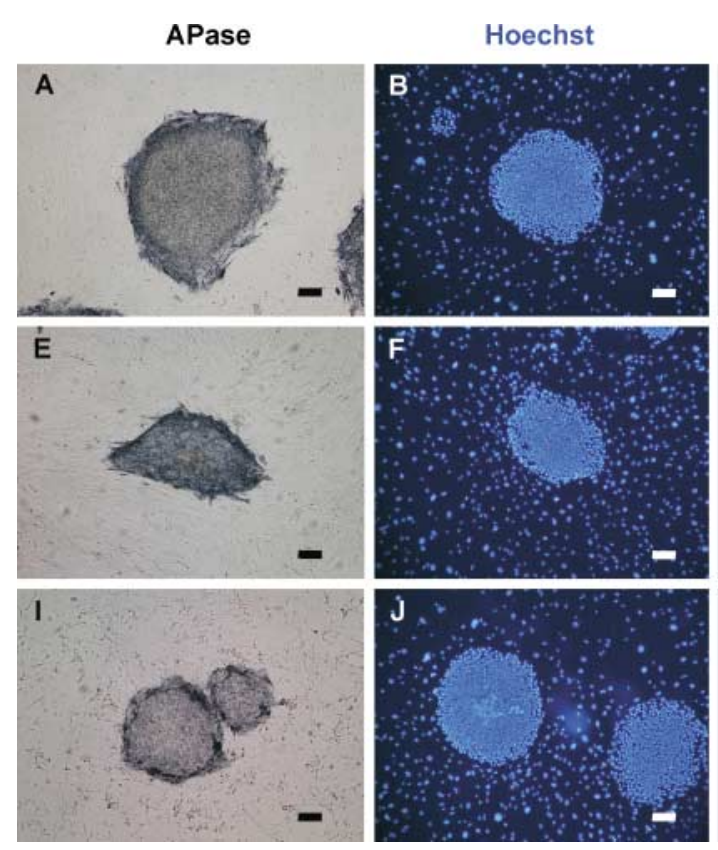

\section{Differentiation capacity of hESCs}

SCID-beige mice were killed 12 weeks after being injected with undifferentiated hESCs that had been cultured on three different human feeder layers. The right testes were injected with hESCs and all showed the formation of teratomas, whereas all the left testes injected only with PBS did not. The tissues formed in the teratomas comprised various types of cells including primitive neuroepithelium (ectoderm), cartilage, renal tissue, tooth-germ (mesoderm) and gastrointestinal epithelium (endoderm) (Fig. 5). hESCs also differentiated spontaneously into various types of cells under the culture condition without feeder and bFGF in vitro. When mechanically harvested hESC colonies maintained on each of the human feeder layers - were cultured in suspension, they formed EBs composed of a heterogeneous population of differentiated cells (Fig. 6A). The expressions of tissue-specific markers related to differentiation were examined by RT-PCR using EBs cultured for 2 weeks. The EBs expressed marker genes of all three embryonic germ layers, including mesoderm (enolase, CMP and kallikrein), endoderm (albumin, $\alpha 1-\mathrm{AT}$ and $\alpha-\mathrm{FP}$ ) and ectoderm (NF-68) (Fig. 6B).

\section{Growth properties of hESCs cultured on each human feeder cell layer}

In the analysis of DNA content, hESCs maintained on MEF or each human feeder layer showed similar proliferation properties. Typically about $10 \%$ of feeder cells (MEF, 9.8\%; hUEC, $10.24 \%$; hBPC, $10.42 \%$; hEF, $10.87 \%$ ) were in the S-phase. However, hESCs contained a higher number of cells in the $S$-phase than feeder cells maintained
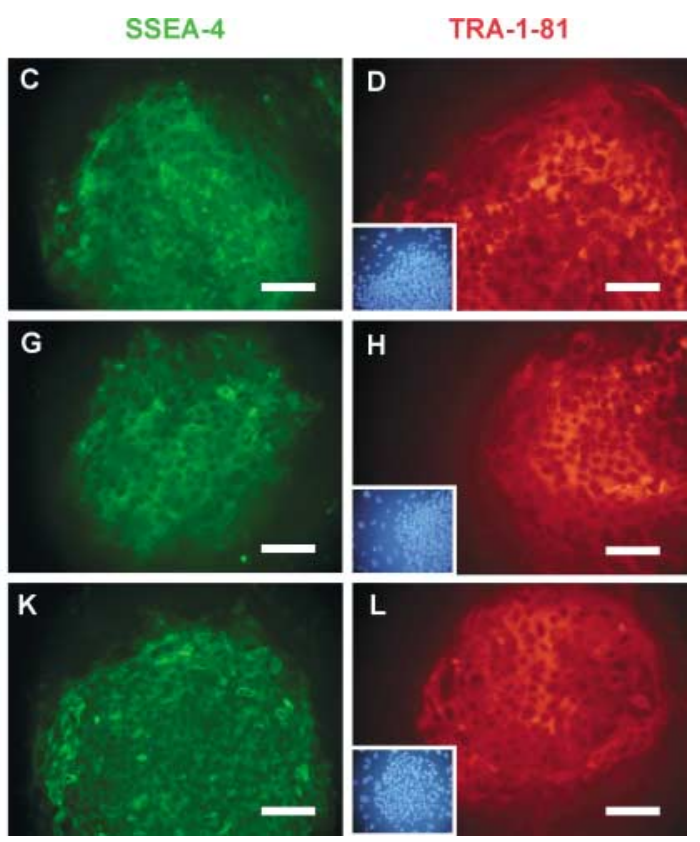

Figure 3 The expression of specific markers from hESCs cultured on each human feeder layer. hESC colonies cultured on hUEC (A-D), hBPC $(E-H)$ and hEF (I-L) feeder cell layers were positive for APase (A, E and I), SSEA-4 (C, G and K) and TRA-1-81 (D, H and L), but negative for SSEA-1 (data not shown). Each feeder cell and hESC was visualized by the staining of Hoechst 33258 (B, F and J). Scale bars represent $100 \mu \mathrm{m}$. 


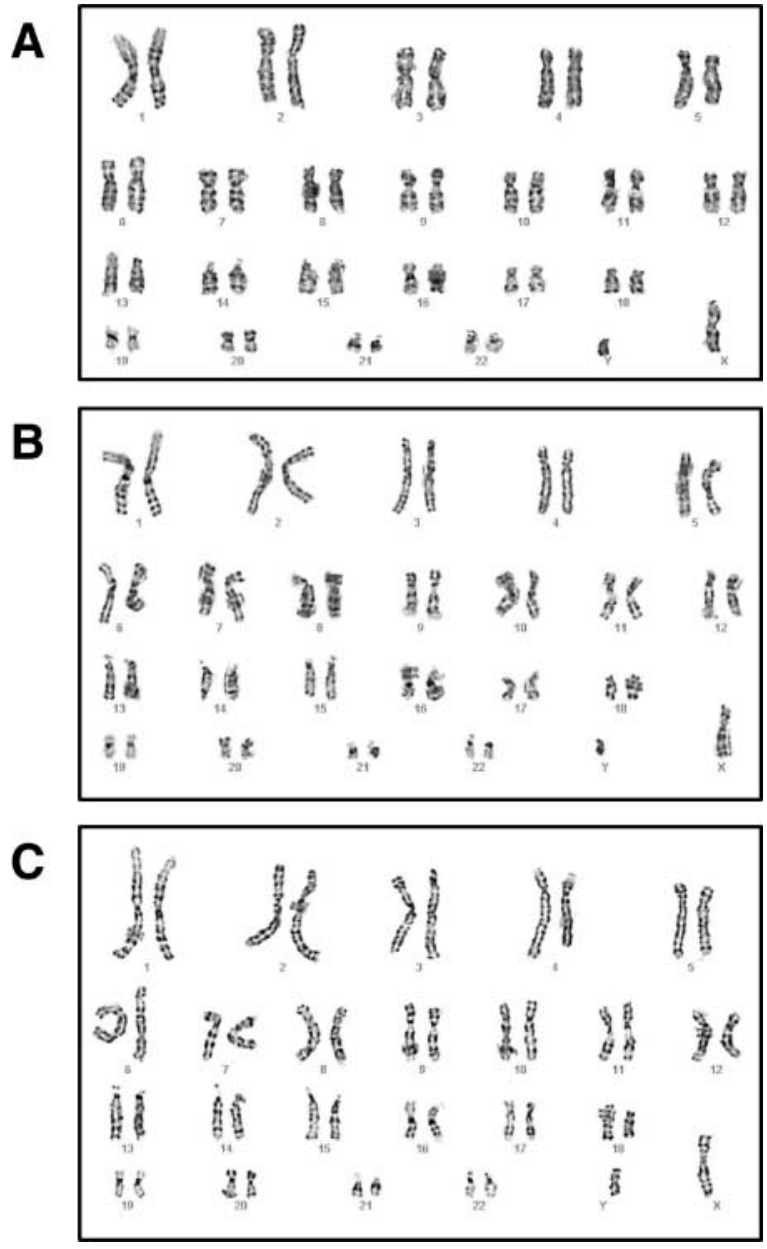

Figure 4 Karyotype analysis of hESCs expanded on each human feeder cell. Miz-hES1 cultured on hUEC (A), hBPC (B) and hEF (C) feeder cells represented normal 46, XY karyotype.

on MEF (48.3\%), hUEC (45.7\%), hBPC (40.2\%) and hEF $(38.7 \%)$ feeder cells respectively (Fig. 7A-H). In addition, MEF- and hUEC-based Miz-hES1 comprised higher numbers of BrdU-incorporated cells than hBPC- and hEFbased hESCs; there were no significant differences however $(P>0.05$; Fig. 7l).

\section{Discussion}

hESCs established and maintained on MEF feeder layers exhibit continuous proliferation without differentiation. However, mouse feeder cells are associated with risks such as viral infection and pathogen transmission when hESCs are used in clinical trials. Various culture conditions have been developed to overcome these problems. Previous studies have used certain types of primary human cells including fetal skin fibroblasts, fallopian-tube epithelial cells, adult marrow cells, foreskin fibroblasts and adult skin fibroblast, as well as commercially available cell lines, as feeder layers (Richards et al. 2002, 2003, Amit et al. 2003, Cheng et al. 2003, Hovatta et al. 2003). One of the improved culture conditions is a feeder-free culture using ECM and MEF-CM or growth cocktail to maintain hESCs in an undifferentiated state $\mathrm{Xu}$ et al. 2001, Amit et al. 2004, Carpenter et al. 2004, Rosler et al. 2004). However, the factors that control self-renewal of hESCs without differentiation remain unclear.

hESCs in an undifferentiated state exhibit a typical morphology of a high nucleus-to-cytoplasm ratio, prominent nucleoli and close spacing between cells. In previous studies the morphology of hESCs maintained on human feeder layers differed slightly from MEF-based hESCs (Amit et al. 2003, Cheng et al. 2003). We also found that the hESC colonies maintained on hUEC and hEF feeder layers were thinner and flatter than MEF- or hBPC-based hESC colonies. On the other hand, hBPC-based hESC colonies were denser than hESCs cultured on other feeder cells such as adult marrow cells (Cheng et al. 2003), and the hESCs (showing slightly different morphology) expressed SSEA-3 and -4, TRA-1-60 and 81, but not SSEA-1. In addition to these markers, proliferation activity was confirmed by the immunocytochemical analysis of APase. As shown in Fig. 3, hESC colonies cultured on each human feeder layer showed typical expression patterns such as being positive for SSEA-4, TRA-1-81 and APase, and negative for SSEA-1. These data indicate that the morphological changes do not affect the expression patterns of pluripotent cell-specific markers. As expected, human-feederbased hESCs also have differentiation capacity; this was confirmed by the formation of teratomas and EBs. Humanfeeder-based hESCs can differentiate into derivatives of all three embryonic germ layers. These results indicate that hESCs maintained on three different human feeder layers exhibited similar morphology, expression of specific markers and differentiation capacity.

hESCs cultured on human feeder layers of hUECs, hBPCs and bEFs were successfully grown as MEF-hESCs. Furthermore, MEF cells can maintain hESCs in an undifferentiated state only until passage 5 , whereas human feeder cells from each of the tissues we tested can be used to support the growth of hESCs until they pass through senescence. This advantage may prove helpful in controlling the growth and differentiation of hESCs that express various embryotrophic factors in each human feeder layer. It is known that hUECs support the growth of cleaving embryos and induce successful implantation. In this process, hUECs express various factors including cell adhesion molecules, growth factors and cytokines that have critical roles in embryo proliferation, attachment and invasion (Aplin 1997, Lessey 2000, Selam et al. 2002, Nardo et al. 2003). Many factors that regulate the growth of cells are also expressed from not only hUECs but also hBPCs (Imagawa et al. 2002, Palmieri et al. 2003) and hEFs (Ellis et al. 1997, Chang et al. 2002). The various factors expressed in each cell type may have an important role in inducing the growth and inhibiting the differentiation of hESCs.

hUECs repeatedly proliferate and differentiate throughout the menstrual cycle, under hormonal regulation. In this experiment, hUECs used to support the growth 

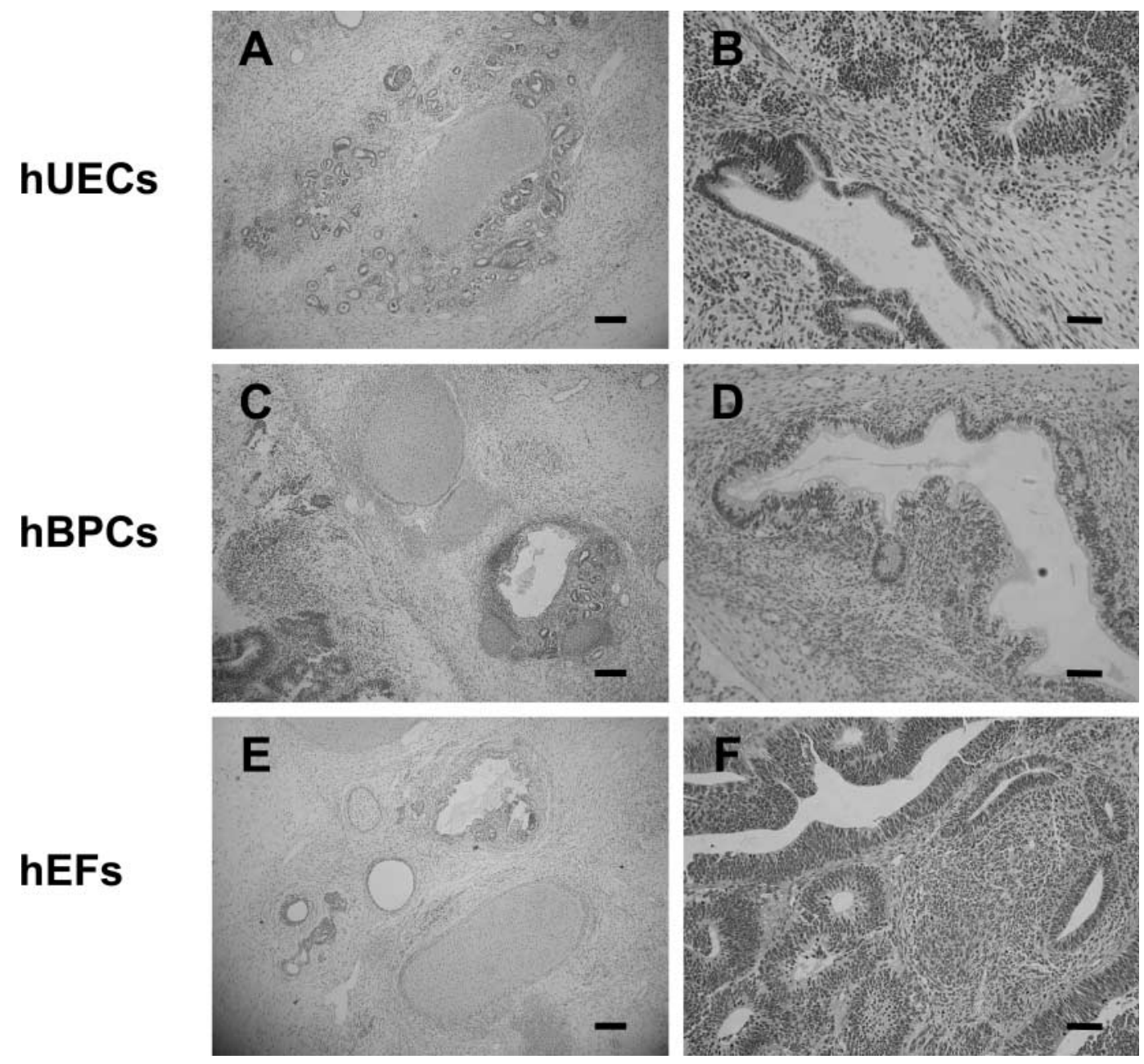

Figure 5 In vivo differentiation (teratoma formation) of hESCs cultured on hUECs (A and B), hBPCs (C and D) and hEFs (E and F). (A) Cartilage and renal tissue, (B) primitive neuroepithelium and epithelial cells, (C) cartilage, renal tissue and primitive neuroepithelium, (D) gastrointestinal epithelium, (E) cartilage, tooth-germ and cystic epithelium, and (F) primitive neuroepithelium. Scale bars represent $100 \mu \mathrm{m}(\mathrm{A}, \mathrm{C}$ and $\mathrm{E})$ and $50 \mu \mathrm{m}(\mathrm{B}, \mathrm{D}$ and $\mathrm{F})$.

A
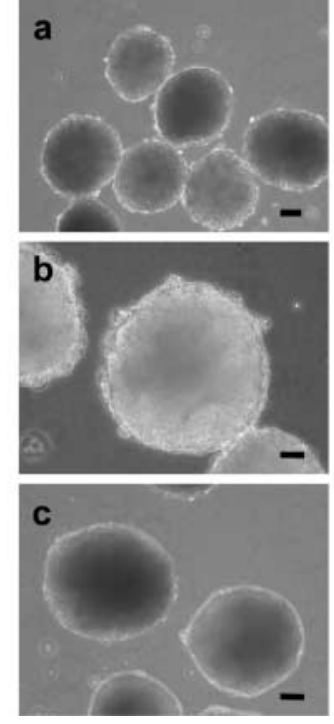

B

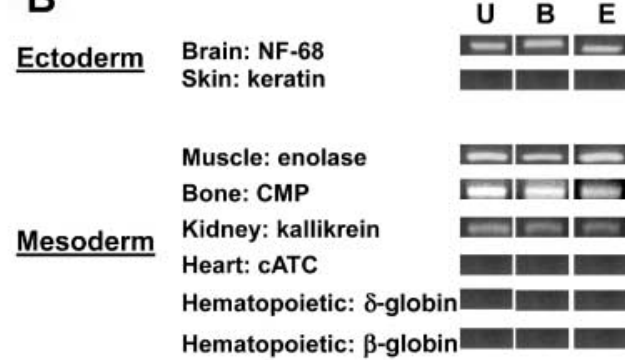

Liver: albumin Liver: $\alpha 1$-antitrypsin

Endoderm Pancreas: PDX-1

Pancreas: insulin

Endoderm: $\alpha-F P$

\section{Control Oct-4}
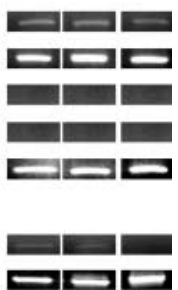

Figure 6 (A) In vitro differentiation (EB formation) of hESCs expanded on hUEC (a), hBPC (b) and hEF (c) feeder layers; scale bars represent $100 \mu \mathrm{m}$ (a and c) and $50 \mu \mathrm{m}$ (b). (B) Expression of tissue-specific marker genes in differentiated EBs. Various marker genes including NF-68 (ectoderm), enolase, CMP, kallikrein (mesoderm), albumin, $\alpha 1-\mathrm{AT}$ and $\alpha$-FP (endoderm) were detected in differentiated EBs derived from $\mathrm{hESCs}$ cultured on each human feeder. U, hUECs; B, hBPCs; E, hEFs. 


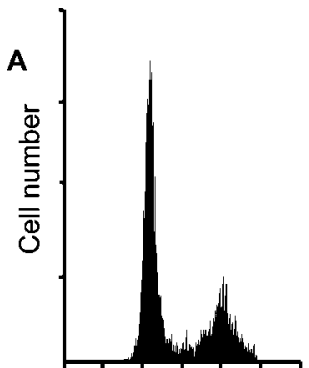

DNA content

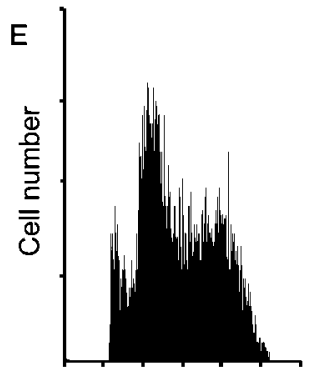

DNA content

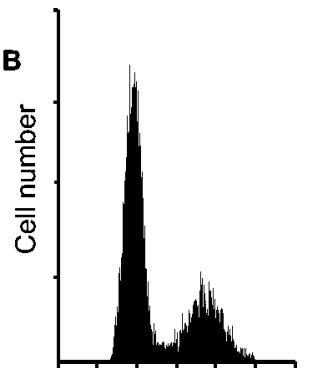

DNA content

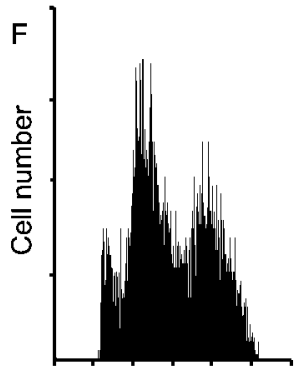

DNA content

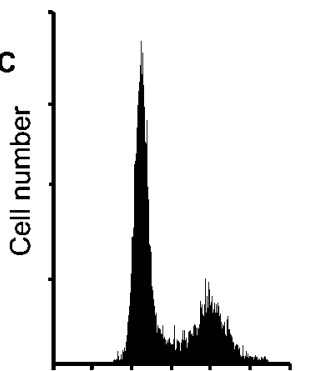

DNA content

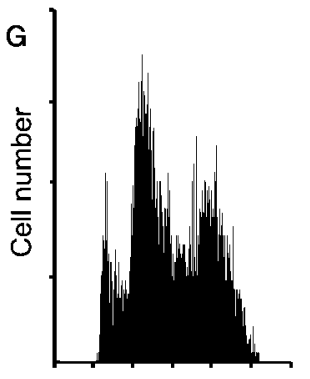

DNA content

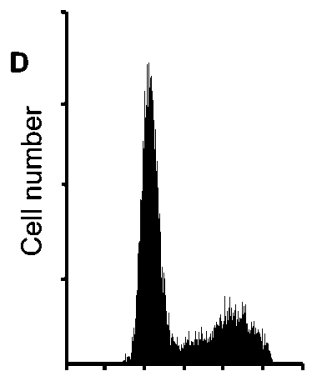

DNA content

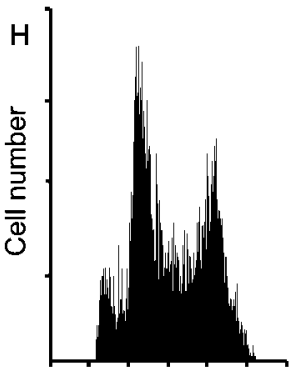

DNA content

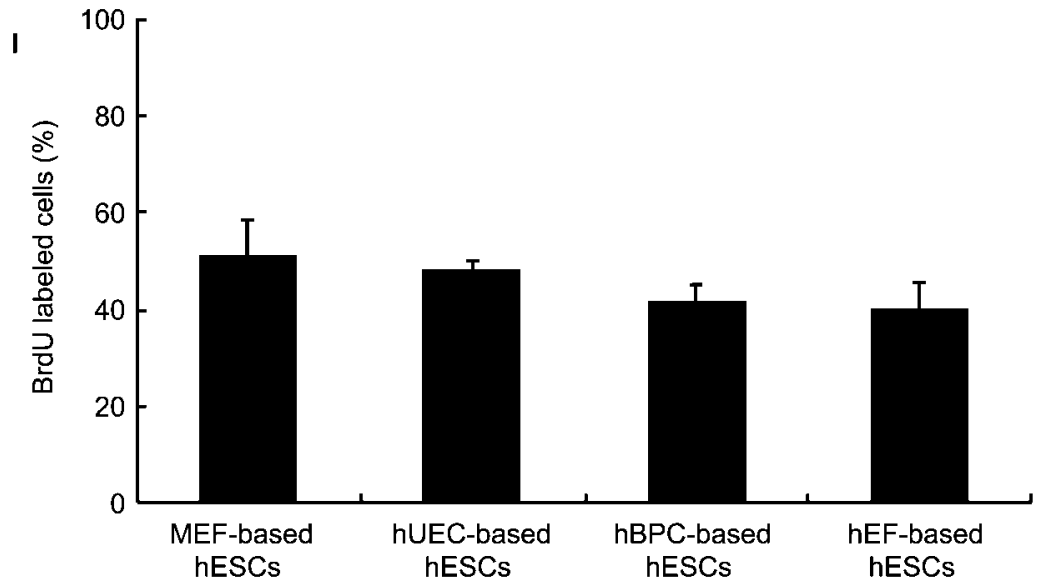

Figure 7 Analysis of DNA contents in each feeder cell $(A-D)$ and feeder-based hESCs $(E-H)$. Human feeder cells such as hUECs (B, 10.24\%), hBPCs (C, $10.42 \%)$ and hEF (D, $10.87 \%)$ showed similar proliferation properties to MEF (A, 9.8\%). MEF(E, 48.3\%) and hUEC-based ( $F, 45.7 \%$ ) hESCs contained higher numbers of proliferating cells than hBPC- (G, 40.2\%) and hEF-based (38.7\%) hESCs. (I) BrdU-incorporated cells showed that MEF- and hUEC-based hESCs have slightly higher proliferation activity than hBPC- and hEF-based hESCs.

of hESCs were obtained from three patients. Two (Miz-endo1 and -2) out of three hUEC lines were isolated at proliferative phase and one cell line (Miz-endo3) was isolated in luteal phase. Mitotically inactivated Mizendo1 and -2, isolated at proliferative phase, can support the continuous growth of hESCs as MEF feeder cells can, but Miz-endo3 cannot. In addition to supporting the maintenance of hESCs, Miz-endo1 can support the establishment of hESC lines. Miz-hES9, -14 and -15 were established and have been expanded on hUEC feeder layers and three hESC lines showed typical hESC properties (Lee et al. 2004). These results suggest that the phase of endometrial cells might be one of the important factors when hUECs are used as feeder cells to keep hESCs in an undifferentiated stage.

For the eventual application in cell replacement therapy, hESCs should be established and maintained under stable xeno-free culture conditions. Thus, many stem cell researchers have tried to eliminate animal materials for the establishment and culture of hESCs. However, the xeno-free culture condition is not yet complete because animal materials have still been used in the process of ICM isolation (anti-human-serum antibody and guinea-pig complement), feeder cell culture (animal serum) and hESC culture (animal serum or SR containing albumin purified from animals). Our results demonstrate that hESCs can be maintained on human feeder cells without direct interaction with animal cells and animal serum. However, our culture condition still has the risks of pathogen transmission and viral infection because FBS was used for the culture of each feeder cell and SR used for the maintenance of hESCs contains bovine albumin. Therefore, it is necessary to establish and culture hESCs using human serum to overcome the contamination of animal resources for cell replacement therapy with a stable xeno-free culture protocol.

More effective human feeder cells should be selected by comparing each type of feeder cell for the expression of 
various factors that are related to the induction of proliferation and the inhibition of differentiation of hESCs - such as ECMs, growth factors and cytokines. hESCs established on the most effective human feeder cells will promote the development of cell-based therapies.

\section{Acknowledgements}

This work was supported by grants (SC12021 and SC11012) from the Stem Cell Research Center of the 21C Frontier R\&D Program, Ministry of Science and Technology, Republic of Korea.

\section{References}

Amit M, Margulets V, Segev H, Shariki K, Laevsky I, Coleman R \& Itskovitz-Eldor J 2003 Human feeder layers for human embryonic stem cells. Biology of Reproduction 68 2150-2156.

Amit M, Shariki C, Margulets V \& Itskovitz-Eldor J 2004 Feeder layer- and serum-free culture of human embryonic stem cells. Biology of Reproduction 70 837-845.

Aplin JD 1997 Adhesion molecules in implantation. Reviews of Reproduction 2 84-93.

Apparao KB, Lovely LP, Gui Y, Lininger RA \& Lessey BA 2002 Elevated endometrial androgen receptor expression in women with polycystic ovarian syndrome. Biology of Reproduction $\mathbf{6 6}$ 297-304.

Carpenter MK, Rosler ES, Fisk GJ, Brandenberger R, Ares X, Miura T, Lucero M \& Rao MS 2004 Properties of four human embryonic stem cell lines maintained in a feeder-free culture system. Developmental Dynamics 229 243-258.

Chang HY, Chi J, Dudoit S, Bondre C, Rijn M, Botstein D \& Brown PO 2002 Diversity, topographic differentiation, and positional memory in human fibroblasts. PNAS 99 12877-12882.

Cheng L, Hammond H, Ye Z, Zhan X \& Dravid G 2003 Human adult marrow cells support prolonged expansion of human embryonic stem cells in culture. Stem Cells 21 131-142.

Ellis I, Banyard J \& Schor SL 1997 Differential response of fetal and adult fibroblasts to cytokines: cell migration and hyaluronan synthesis. Development 124 1593-1600.

Evans MJ \& Kaufman MH 1984 Establishment in culture of pluripotential cells from mouse embryos. Nature 292 154-156.

Hahm HA \& Ip MM 1990 Primary culture of normal rat mammary epithelial cells within a basement membrane matrix. I. Regulation of proliferation by hormones and growth factors. In Vitro Cell Developmental Biology 26 791-802.

Hovatta O, Mikkola M, Gertow K, Strömberg A, Inzunza J, Hreinsson J, Rozell B, Andäng M \& Ährlund-Richter L 2003 A culture system using human foreskin fibroblasts as feeder cells allows production of human embryonic stem cells. Human Reproduction $\mathbf{1 8}$ 1404-1409.

Imagawa W, Pedchenko VK, Helber J \& Zhang H 2002 Hormone/ growth factor interactions mediating epithelial/stromal communication in mammary gland development and carcinogenesis. Journal of Steroid Biochemistry and Molecular Biology $\mathbf{8 0}$ $213-230$.

Lee PP, Hwang JJ, Murphy G \& Ip MM 2000 Functional significance of MMP-9 in tumor necrosis factor-induced proliferation and branching morphogenesis of mammary epithelial cells. Endocrinology 141 3764-3773.

Lee JB, Lee JE, Park JH, Kim SJ, Kim MK, Roh SI \& Yoon HS 2004 Establishment and maintenance of human embryonic stem cell lines on human feeder cells derived from uterine endometrium under serum-free condition. Biology of Reproduction (In Press).

Lessey BA 2000 The role of the endometrium during embryo implantation. Human Reproduction Supplement 6 39-50.
Nardo LG, Nikas G \& Makrigiannakis A 2003 Molecules in blastocyst implantation. Role of matrix metalloproteinases, cytokines and growth factors. Journal of Reproductive Medicine 48 137-147.

Palmieri C, Roberts-Clark D, Assadi-Sabet A, Coope RC, O'Hare M, Sunters A, Hanby A, Slade MJ, Gomm JJ, Lam EW \& Coombes RC 2003 Fibroblast growth factor 7, secreted by breast fibroblasts, is an intereukin-1 beta-induced paracrine growth factor for human breast cells. Journal of Endocrinology 177 65-81.

Park JH, Kim SJ, Oh EJ, Moon SY, Roh SI \& Yoon HS 2003 Establishment and maintenance of human embryonic stem cells on STO, a permanently growing cell line. Biology of Reproduction 69 2007-2014.

Reubinoff BE, Pera MF, Fong CY, Trounson A \& Bongso A 2000 Embryonic stem cell lines from human blastocysts: somatic differentiation in vitro. Nature Biotechnology 18 399-404.

Ricciardelli C, Brooks JH, Suwiwat S, Sakko AJ, Mayne K, Raymond WA, Seshadri R, LeBaron RG \& Horsfall DJ 2002 Regulation of stromal versican expression by breast cancer cells and importance to relapse-free survival in patients with nodenegative primary breast cancer. Clinical Cancer Research 8 1054-1060.

Richards M, Fong CY, Chan WK, Wong PC \& Bongso A 2002 Human feeders support prolonged undifferentiated growth of human inner cell masses and embryonic stem cells. Nature Biotechnology 20 933-936.

Richards M, Tan S, Fong CY, Biswas A, Chan WK \& Bongso A 2003 Comparative evaluation of various human feeders for prolonged undifferentiated growth of human embryonic stem cells. Stem Cells 21 546-556.

Rosler ES, Fisk GJ, Ares X, Irving J, Miura T, Rao MS \& Carpenter MK 2004 Long-term culture of human embryonic stem cells in feeder-free conditions. Developmental Dynamics 229 $259-274$.

Roy A, Krzykwa E, Lemieux R \& Neron S 2001 Increased efficiency of $\gamma$-irradiated versus mitomycin C-treated feeder cells for the expansion of normal human cells in long-term cultures. Journal of Hematotherapy and Stem Cell Research 10 873-880.

Selam B, Kayisli UA, Garcia-Valasco JA \& Arici A 2002 Extracellular matrix-dependent regulation of Fas ligand expression in human endometrial stromal cells. Biology of Reproduction $\mathbf{6 6}$ $1-5$.

Smith AG, Heath JK, Donaldson DD, Wong GG, Moreau J, Stahl M \& Rogers D 1988 Inhibition of pluripotential embryonic stem cell differentiation by purified polypeptides. Nature 336 688-690.

Telgmann R, Maronde E, Tasken K \& Gellersen B 1997 Activated protein kinase $\mathrm{A}$ is required for differentiation-dependent transcription of the decidual prolactin gene in human endometrial stromal cells. Endocrinology 138 929-937.

Thomson JA, Itskovitz-Eldor J, Shapiro SS, Waknitz MA, Swiergiel JJ, Marshall VS \& Jones JM 1998 Embryonic stem cell lines derived from human blastocysts. Science 282 1145-1147.

Van Antwerp DJ, Martin SJ, Kafri T, Green DR \& Verma IM 1996 Suppression of TNF-alpha-induced apoptosis by NF-kappaB. Science 274 787-789.

Williams RL, Hilton DJ, Pease S, Willson TA, Stewart CL, Gearing DP, Wagner EF, Metcalf D, Nicola NA \& Gough NM 1988 Myeloid leukemia inhibitory factor maintains the developmental potential of embryonic stem cells. Nature 336 684-687.

Xu C, Inokuma MS, Denham J, Golds K, Kundu P, Gold JD \& Carpenter MK 2001 Feeder-free growth of undifferentiated human embryonic stem cells. Nature Biotechnology 19 971-974.

Received 8 July 2004

First decision 3 August 2004

Revised manuscript received 23 August 2004

Accepted 3 September 2004 